\title{
ARMA Modeling via Bounded-Real Functions and Lattice Filters
}

\author{
Jingwu Yao, and Sueo Sugimoto \\ Department of Electrical and Electronic Engineering, Ritsumeikan University \\ Noji, Kusatsu City, Shiga 525-77 Japan \\ fee30029@bkc.ritsumei.ac.jp, and sugimoto@bkc.ritsumei.ac.jp
}

\begin{abstract}
The identification problem for ARMA models is considered without a prior $i$ knowledge of either numerator or denominator degress, from a new view-point based on bounded-real functions and lattice filters. Using Pillai's algorithm, which is founded on the powerful concepts in passive networks, we obtain the estimation of the spectral density of an ARMA process. A lattice whitening filter is used to generate the error filter. This allows us to develop and justify a new scheme for ARMA model order determination as well as parameter estimation. It is shown that the proposed method is more satisfactory especially for short data records by simulation experiments.
\end{abstract}

\section{Introduction}

Autoregressive (AR) and autoregressive moving average (ARMA) models have been extensively investigated in a variety of fields, such as statistics, engineering, econometrics and seismology. Therefore, numerous algorithms utilizing these models have been developed[4]. In this paper, we consider the identification problem for ARMA models from a finite set of observations. A new method based on bounded-real functions and lattice filters is proposed.

It is well known that the techniques for AR parameter estimation are intimately related to the Yule-Walker equations, which calculate the linear prediction coefficients of the AR precess from the autocovariance functions. And the Yule-Walker equations can be solved recursively by the Levinson-Durbin algorithm. The lattice structure filter, which may be interpreted as a realization of the Levinson-Durbin's algorithm, has many advantageous properties and has found wide application in signal processing, spectral estimation, speech analysis, etc..

On the other hand, positive-real functions (p.r.) and bounded-real functions (b.r.), an indispensable tool in classical network analysis and synthesis problems, are well understood. Positive-real functions and boundedreal functions have been used to study adaptive system control, $H_{\infty}$ control and nonlinear control[10]. It is clear that the powerful concepts of p.r. functions and b.r. functions are of fundamental importance in system stability analysis. And their intimate one-toone connection with power spectra makes it possible to study many signal processing problems from a new viewpoint [2]. Recently, by using the deterministic theory founded on p.r. functions and b.r. functions, Pillai et al.[1] have proposed a new technique for spectral estimation and ARMA-system identification from the given autocovariance functions, which is also extended to multichannel system identification and multichannel rational approximation by themselves[2]. In our previous works[7][8], we proposed one improved criterion for ARMA model order determination based on Pillai's method, and considered the algorithm for noisy AR processes. But in general, the exact autocovariance functions $\left\{c_{r},|r|=0 \rightarrow \infty\right\}$ are not known from available measurable ARMA data, such that we have to replace the $c_{r}$ 's by their estimates $\hat{c}_{r}$ 's. Thus these approaches in [1], [2], [7], [8] are not very satisfactory to estimate ARMA models, especially for short data records. For obtaining the spectral estimation of an ARMA process, here we derive the lattice filter to estimate the error filter from a segment of an observed record, which is different from Pillai's algorithm. This allows us to develop and justify a new scheme for ARMA model order determination as well as parameter estimation.

\section{Preliminaries}

\subsection{ARMA Models}

Consider the following $\operatorname{ARMA}(p, q)$ process $x(n)$,

$$
x(n)=-\sum_{i=1}^{p} \alpha_{i} x(n-i)+\sum_{i=0}^{q} \beta_{i} \omega(n-i),
$$

where $\omega(n)$ denotes a zero-mean, real second-order stationary white noise process of unit variance. Because $x(n)$ is a wide-sense stationary stochastic process with finite power, the spectral density $S_{x}(\theta)$ of $x(n)$ is periodic with period $2 \pi$,

$$
\int_{-\pi}^{\pi} S_{x}(\theta) d \theta<\infty
$$


and is also satisfies the Paley-Wiener criterion

$$
\int_{-\pi}^{\pi} \ln S_{x}(\theta) d \theta>-\infty
$$

Denote the autocovariance functions of the series $\{x(n)\}$ by $\left\{c_{r}\right\}$ where

$$
c_{r} \equiv E[x(n) x(n+r)]=c_{-r}, \quad|r|=0 \rightarrow \infty,
$$

then, the spectral density is given by

$$
S_{x}(\theta)=\sum_{r=-\infty}^{\infty} c_{r} e^{j r \theta} \geq 0
$$

The nonnegativity property of the spectral density can be characterized in terms of the nonnegativity of every Toeplitz matrix $\mathbf{T}_{r}$ generated from its autocovariances. i.e., $S_{x}(\theta) \geq 0 \Leftrightarrow \mathbf{T}_{r} \geq 0, r=0 \rightarrow \infty$, where

$$
\mathbf{T}_{r}=\left[\begin{array}{cccc}
c_{0} & c_{1} & \ldots & c_{r} \\
c_{-1} & c_{0} & \ldots & c_{r-1} \\
\vdots & \vdots & \ddots & \vdots \\
c_{-r} & c_{-r+1} & \ldots & c_{0}
\end{array}\right]
$$

In addition, if $S_{x}(\theta)$ also satisfies the Paley-Wiener criterion, the nonnegativity property of the spectral density implies positive definiteness for every $\mathbf{T}_{r}$ in (6). i.e., $S_{x}(\theta) \geq 0 \Rightarrow \mathrm{T}_{r}>0, r=0 \rightarrow \infty$.

Under these conditions (2) and (3), ARMA spectral density $S_{x}(\theta)$ can be expressed as

$$
S_{x}(\theta)=\left|H\left(e^{j \theta}\right)\right|^{2}=\sum_{r=-\infty}^{\infty} c_{r} e^{j r \theta} .
$$

$H(z)$ is called minimum-phase and stable,

$$
H(z)=\frac{B(z)}{A(z)}=\frac{\beta_{0}+\beta_{1} z+\cdots+\beta_{q} z^{q}}{1+\alpha_{1} z+\cdots+\alpha_{p} z^{p}}, \quad p \geq q
$$

represents the transfer function of ARMA model. The goal of this paper is to evaluate the model order $p$, $q$ as well as the system parameters $\left\{\alpha_{i}, i=1 \rightarrow p\right\}$ and $\left\{\beta_{i}, i=0 \rightarrow q\right\}$ from the available observations $\{x(n), n=1 \rightarrow N\}$.

\subsection{P.R Functions, B.R. Functions and ARMA Characterization[1][2]}

The concepts of p.r. functions and b.r. functions are best illustrated in classical network theory. The impedance function $Z(z)$ associated with every passive network is shown to be a p.r. function. Also, the physical meaning of b.r. functions is not difficult to understand. If we introduce an incident wave $w_{1}(z)$ and a reflected wave $w_{2}(z)$ at the impedance terminals to symbolically represent the incoming and outgoing power carriers, then the reflection coefficient represents a b.r. function $\rho(z)$, i.e., $\rho(z)=w_{2}(z) / w_{1}(z)$. There exists a one-to-one relationship between a p.r. function and b.r. function, i.e.,

$$
\rho(z)=\frac{Z(z)-R_{0}}{Z(z)+R_{0}^{*}}, \quad \operatorname{Re} R_{0}>0 .
$$

Consider the following power series expansion

$$
Z(z)=c_{0}+2 \sum_{r=1}^{\infty} c_{r} z^{r}, \quad|z|<1
$$

then $Z(z)$ represents a p.r. function due to Schur's theorem[2], which is that $Z(z)$ given by (10) defines a p.r. function iff every Toeplitz matrix $\mathbf{T}_{r}$ generated from $\left\{c_{r}, r=0 \rightarrow \infty\right\}$ as in (6) is nonnegative definite. Thus, there exists a one-to-one relationship between p.r. function and power spectral density. Since every power spectral density can be represented uniquely in terms of its minimum phase factor $H(z)$, then for an $\operatorname{ARMA}(p, q)$ process $x(n)$ as in (1), we conclude that, there exists a one-to-one relationship between p.r. function and ARMA model. The relationship is given by

$$
\frac{Z(z)+Z_{*}(z)}{2}=H(z) H_{*}(z)=z^{p-q} \cdot \frac{B(z) \tilde{B}(z)}{A(z) \tilde{A}(z)}
$$

where $H_{*}(z) \equiv H^{*}\left(1 / z^{*}\right)=H(1 / z), \tilde{A}(z) \equiv z^{p} A_{*}(z)$, $\tilde{B}(z) \equiv z_{q} B_{*}(z)$. Such a $Z(z)$ is free of poles on $|z|=1$ and of degree $\delta(Z(z))=\max (p, q)=p$, and hence it can be simplified into

$$
Z(z)=\frac{d_{0}+d_{1} z+\cdots+d_{p-1} z^{p-1}+d_{p} z^{p}}{1+\alpha_{1} z+\cdots+\alpha_{p-1} z^{p-1}+\alpha_{p} z^{p}} .
$$

Considering the cascade representation problem[5] for every such p.r. function $Z(z)$ based on the Schur algorithm[2], we have a network shown in Fig. 1. Here we have

$$
\rho_{0}(z) \equiv \frac{Z(z)-Z(0)}{Z(z)+Z(0)}, \quad|z|<1
$$

is a b.r.function, and the single-step update rule (Schur algorithm) is given by

$$
\rho_{r}(z)=\frac{z \rho_{r+1}(z)+s_{r}}{1+z s_{r} \rho_{r+1}(z)}, \quad r>0
$$

where $s_{r}=\rho_{r}(0)$ represent the $(r)$ st junction "mismatch" reflection coefficient.

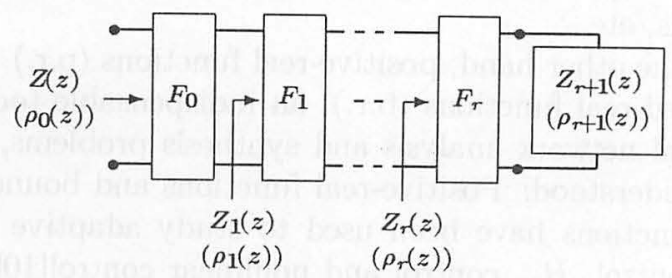

Fig. 1. Cascade representation 
In Fig.1, the p.r. function in (12) has been realized as the input impedance of a cascade network containing a set of ideal line elements and an irreducible p.r. function. Notice that $z=0$ is a zero of $\left(Z(z)+Z_{*}(z)\right) / 2$ of exact order $p-q$ in (11). By using Richards's theorem[5] on degree reduction, we can obtain

$$
\begin{array}{r}
\delta\left(\rho_{r}(z)\right)=\delta\left(Z_{r}(z)\right)=p-r, \\
\text { for } \quad r=0 \rightarrow p-q-1,
\end{array}
$$

and the degree constraint

$$
\begin{aligned}
& \delta\left(\rho_{r+1}(z)\right)=\delta\left(z \rho_{r+1}(z)\right) \\
& \quad=\delta\left(Z_{r}(z)\right)=q, \quad \text { for } \quad r \geq p-q .
\end{aligned}
$$

As a result[1][2], for any $r \geq p-q$, every such $\rho_{r+1}(z)$ is expressible in irreducible form as the ratio of a numerator polynomial of degree $\leq q-1$ and a denominator polynomial of degree equal to $q$,

$$
\begin{aligned}
\rho_{r+1}(z) & =\frac{\left.h_{(} z\right)}{\left.g_{(} z\right)} \\
= & \frac{h_{0}+h_{1} z+\cdots+h_{q-1} z^{q-1}}{1+g_{1} z+\cdots+g_{q-1} z^{q-1}+g_{q} z^{q}} .
\end{aligned}
$$

Equation (15) and (16) represent the key feature of $\operatorname{ARMA}(p, q)$ system $H(z)$ as in (8). This invariant character of the b.r. functions with respect to their degree beyond a certain stage is exploited to identify the correct model order and system parameters.

\section{ARMA Spectral Estimation}

Using Youla's theorem[1][2], the spectrum of ARMA process $x(n)$ is given by

$$
\begin{aligned}
S_{x}(\theta) & =\left|H\left(e^{j \theta}\right)\right|^{2} \\
= & \frac{1-\left|\rho_{r+1}\left(e^{j \theta}\right)\right|^{2}}{\left|P_{r}\left(e^{j \theta}\right)-e^{j \theta} \rho_{r+1}\left(e^{j \theta}\right) \tilde{P}_{r}\left(e^{j \theta}\right)\right|^{2}},
\end{aligned}
$$

where $\rho_{r+1}(z)$ is an arbitrary b.r. fucntion that represents the termination after $r+1$ line extractions in Fig.1, $P_{r}(z)$ is a Levinson polynomial of degree $r$ obtained by

$$
\begin{aligned}
& P_{r}(z)=\frac{1}{\sqrt{\Delta_{r-1} \Delta_{r}}} \operatorname{det}\left[\begin{array}{ccc|c} 
& & c_{r} \\
\mathrm{~T}_{r-1} & \vdots \\
& & c_{1} \\
\hline z^{r} & \cdots & z & 1
\end{array}\right] \\
& \equiv k_{0}+k_{1} z+\cdots+k_{r} z^{r}, \quad r \geq 0 \text {, }
\end{aligned}
$$

$\mathrm{T}_{r}$ represents the Toeplitz matrix in (6), $\Delta_{r}=\operatorname{det} \mathbf{T}_{r}$ $\left(\Delta_{-1} \equiv 1, P_{0}(z) \equiv 1 / \sqrt{c_{0}}\right)$, and $\tilde{P}_{r}(z)$ is its reciprocal polynomial $\left(\tilde{P}_{r}(z) \equiv z^{r} P_{r}(1 / z)\right)$. Thus we can state ARMA spectral estimation in (18) as the determination of a b.r. function $\rho_{r+1}(z)$ by solving a Levinson polynomial $P_{r}(z)$ from $\left\{c_{k},|k|=0 \rightarrow r\right\}$. In [1], [2], based on Schur algorithm in (14), the recursion of the polynomial $P_{r}(z)$ is given by

$$
\begin{aligned}
& \sqrt{1-s_{r}^{2}} P_{r}(z)=P_{r-1}(z)-z s_{r} \tilde{P}_{r-1}(z), \\
& \quad r=1 \rightarrow \infty
\end{aligned}
$$

that begins under the initialization $P_{0}(z)=1 / \sqrt{c_{0}}$. And from a fundamental matrix identity[2] applied to $\Delta_{r}$, a new formula is also given by

$$
\frac{\Delta_{r}}{\Delta_{r-1}}=\frac{\Delta_{r-1}}{\Delta_{r-2}}\left(1-\left|s_{r}\right|^{2}\right),
$$

where the junction reflection coefficients satisfy

$$
s_{r}=(-1)^{r-1} \frac{\Delta_{r}^{(1)}}{\Delta_{r-1}},
$$

and $\Delta_{r}^{(1)}$ represents the minor of $\mathbf{T}_{r}$ obtained after deleting its first column and last row.

Here we begin from linear prediction to give a latticebased approach for solving the Levinson polynomial $P_{r}(z)$.

Consider a finite power discrete-time regular stochastic process $x(n)$ with power spectral density $S_{x}(\theta)$ that satisfies (2) and (3). We form the sum

$$
\hat{x}_{r}(n)=-\sum_{i=1}^{r} a_{i, r} x(n-i)
$$

in terms of its $r$ most past values (forward predictor) as the estimate of $x(n)$. The coefficients $a_{i, r}$ are so chosen as to minimize the MS value of the resulting error $\hat{\varepsilon}_{r}(n)=x(n)-\hat{x}_{r}(n)$. The error $\hat{\varepsilon}_{r}(n)$ is the output of the forward error filter

$$
E_{r}(z)=1+\sum_{i=1}^{r} a_{i, r} z^{i}
$$

with input $x(n)$. Now we denote by $\check{x}_{r}(n)$ the estimate of $x(n)$ in terms of its $r$ most recent future values (backward predictor). We can conclude that the backward predictor of $x(n)$ equals

$$
\check{x}_{r}(n)=-\sum_{i=1}^{r} a_{i, r} x(n+i),
$$

where the coefficients $a_{i, r}$ are the same as in (24). The backward error filter is given by

$$
E_{r}\left(z^{-1}\right)=1+\sum_{i=1}^{r} a_{i, r} z^{-i} .
$$

Its response to $x(n)$ equals the backward error $\breve{\varepsilon}_{r}(n)=$ $x(n)-\check{x}_{r}(n)$. As well known, the predictor of $x(n)$ can be determined recursively by Levinson-Durbin's algorithm[3]

$$
\left\{\begin{array}{l}
\hat{\varepsilon}_{r}(n)=\hat{\varepsilon}_{r-1}(n)-K_{r} \breve{\varepsilon}_{r-1}(n-r) \\
\breve{\varepsilon}_{r}(n-r)=\breve{\varepsilon}_{r-1}(n-r)-K_{r} \hat{\varepsilon}_{r-1}(n)
\end{array},\right.
$$




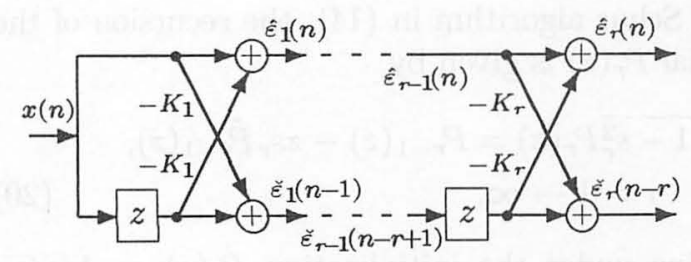

Fig. 2. Lattice filter

where $K_{r}$ is a constant to be determined, and is also called the reflection cofficient (PARCOR parameters). Now we rewrite Levinson-Durbin's algorithm as follows,

$$
\left\{\begin{array}{l}
a_{i, r}=a_{i, r-1}-K_{r} a_{r-i, r-1}, \quad i=1 \rightarrow r-1 \\
a_{r, r}=-K_{r}
\end{array}\right.
$$

and

$$
\begin{aligned}
\sigma_{r-1}^{2} K_{r} & =c_{r}+\sum_{i=1}^{r-1} a_{i, r-1} c_{r-i}, \\
\sigma_{r}^{2} & =\sigma_{r-1}^{2}\left(1-K_{r}^{2}\right),
\end{aligned}
$$

where $\sigma_{r}^{2}$ represents the MS error at the $(r)$ th stage. The iteration starts with $\sigma_{0}^{2}=E\left[x^{2}(n)\right]=c_{0}$. In Fig.2, we show a lattice filter structure. The system function from the input to the upper output equals $E_{r}(z)$, and from the input to the lower output it equals $z^{r} E_{r}\left(z^{-1}\right)$. This yields

$$
\left\{\begin{array}{l}
E_{r}(z)=E_{r-1}(z)-K_{r} z^{r} E_{r-1}\left(z^{-1}\right) \\
z^{r} E_{r}\left(z^{-1}\right)=z^{r} E_{r-1}\left(z^{-1}\right)-K_{r} E_{r-1}(z)
\end{array} .\right.
$$

In the following theorem, we give a relationship between Pillal's algorithm and the lattice-based approach.

Theorem 1 Under the assumption of known secondorder statistics;

1) The $(r)$ st junction "mismatch" reflection coefficient in Schur algorithm is equal to the $(r)$-stage PARCOR parameter of a lattice filter, i.e.,

$$
\rho_{r}(0)=s_{r}=K_{r}, \quad r=1 \rightarrow \infty .
$$

2) The Levinson polynomial $P_{r}(z)$ defined in (19) is equivalent to the error filter $E_{r}(z)$ in linear prediction, and can be written as

$$
P_{r}(z)=\frac{1}{\sqrt{\sigma_{r}^{2}}} \cdot E_{r}(z), \quad r=1 \rightarrow \infty ;
$$

Proof. The MS error $\sigma_{r}^{2}$ can be calculated by the formula[3]

$$
\sigma_{r}^{2}=\frac{\Delta_{r}}{\Delta_{r-1}} .
$$

We substitute (34) into (30) and compare it with (21). This yields $s_{r}=K_{r}$.

Substituting (34) into (21), we obtain

$$
\sqrt{1-s_{r}^{2}}=\sqrt{\frac{\sigma_{r}^{2}}{\sigma_{r-1}^{2}}} .
$$

From (35) and (20) this follows that

$$
\begin{aligned}
\sqrt{\sigma_{r}^{2}} \cdot P_{r}(z) & =\sqrt{\sigma_{r-1}^{2}} \cdot P_{r-1}(z) \\
& -K_{r} z^{r} \sqrt{\sigma_{r-1}^{2}} \cdot P_{r-1}\left(z^{-1}\right)
\end{aligned}
$$

for $r=1 \rightarrow \infty$. Comparing (31) with (36), we can complete the proof for (33).

Now substituting (33) into (18), we conclude that the class of all spectral extension for every $r \geq 0$ is given by

$$
S_{x}(\theta)=\frac{\sigma_{r}^{2} \cdot\left[1-\left|\rho_{r+1}\left(e^{j \theta}\right)\right|^{2}\right]}{\left|E_{r}\left(e^{j \theta}\right)-e^{j \theta} \rho_{r+1}\left(e^{j \theta}\right) \tilde{E}_{r}\left(e^{j \theta}\right)\right|^{2}} .
$$

In fact, Papoulis[3], using the development on Levinson's algorithm and the equivalence between Hurwitz polynomials, reflection coefficients, and positive definite sequences, gave the same result as in (37). However in [3], more information about b.r. function $\rho_{r+1}(z)$ was not discussed. In next section, we exploit the characterization of b.r. function $\rho_{r+1}(z)$ discussed in Section 2 to complete the ARMA identification problem, which is based on Pillai's algorithm.

\section{ARMA Modeling Algorithm}

As above shown in subsection 2.1,

$$
S_{x}(\theta)=\left|H\left(e^{j \theta}\right)\right|,
$$

where $H(z)$ is also unique up to sign, and

$$
H(z)=\frac{B(z)}{A(z)}=\frac{\sqrt{\sigma_{r}^{2}} \cdot \psi_{r+1}(z)}{g(z) E_{r}(z)-z h(z) \tilde{E}_{r}(z)}
$$

is the unique minimum phase rational factor associated with $S_{x}(\theta)$ in (37), since $H(z)$ is analytic together with its inverse in $|z|<1$. And $H(z)$ represents a basic interpolatory formula valid for all $r \geq 0$. Here $\psi_{r+1}(z)$ is the unique anti-Schur polynomial solution of the equation

$$
\psi_{r+1}(z) \psi_{r+1 *}(z)=g(z) g_{*}(z)-h(z) h_{*}(z) .
$$

For the case of given data $\{x(n)\}$, it is known that the lattice algorithms confirm stability and have good numerical properties. In our proposed scheme, all estimates of the error filter $E_{r}(z)$ in (38) are obtained using the Burg's algorithm[4]

$$
K_{r}=\frac{2 \sum_{n=r}^{N-1} \hat{\varepsilon}_{r-1}(n) \check{\varepsilon}_{r-1}(n-1)}{\sum_{n=r}^{N-1}\left[\hat{\varepsilon}_{r-1}(n)^{2}+\check{\varepsilon}_{r-1}(n-1)^{2}\right]},
$$

with $\hat{\varepsilon}_{0}(n)=\check{\varepsilon}_{0}(n)=x(n)$. From (30), the MS error $\sigma_{r}^{2}$ in (38) is given by

$$
\sigma_{r}^{2}=\prod_{i=1}^{r}\left(1-K_{i}^{2}\right) \sigma_{0}^{2}=\prod_{i=1}^{r}\left(1-K_{i}^{2}\right) c_{0} .
$$


After estimate the error filter $E_{r}(z)$, the next is similar to the method in [7]. As the summary, we describe the scheme as follows.

For determining the model order $p$ and $q$ in (8), the sequential procedure is started with $n=1, m=0$ and $m \leq n$ to formalize the model order selection. Recall [7], that the criterion for ARMA model order determination is given by

$$
\epsilon \equiv \mathbf{B}\left(\mathbf{B}^{T} \mathbf{B}\right)^{-1} \mathbf{B}^{T} \mathbf{c}-\mathbf{c},
$$

and

$$
\epsilon^{T} \epsilon \approx 0,
$$

whenever $n \geq p$ and $m=q$, where $\mathbf{B}$ and $\mathrm{c}$ are a $(2 m+1) \times 2 m$ matrix and a $(2 m+1) \times 1$ matrix, respectively, given by the coefficients $b_{k}$ 's of the error filter $E_{n+m+1}(z)$, i.e.,

$$
\begin{aligned}
& \mathbf{B} \equiv \\
& {\left[\begin{array}{cccccc}
b_{n} & \ldots & b_{n-m+1} & -b_{m+1} & \ldots & -b_{2 m} \\
b_{n+1} & \ldots & b_{n-m+2} & -b_{m} & \ldots & -b_{2 m-1} \\
\vdots & \ldots & \vdots & \vdots & \ldots & \vdots \\
b_{n+m-1} & \ldots & b_{n} & -b_{2} & \ldots & -b_{m+1} \\
b_{n+m} & \ldots & b_{n+1} & -b_{1} & \ldots & -b_{m} \\
b_{n+m+1} & \ldots & b_{n+2} & -1 & \ldots & -b_{m-1} \\
\vdots & \ldots & \vdots & \vdots & \ldots & \vdots \\
0 & \ldots & b_{n+m+1} & 0 & \ldots & -1
\end{array}\right]}
\end{aligned}
$$

$$
\mathrm{c} \equiv-\left[\begin{array}{llllllll}
b_{n+1} & b_{n+2} & \ldots & b_{n+m+1} & 0 & 0 & \ldots & 0
\end{array}\right]^{T} \text {. }
$$

Since the first stage where (43) is satisfied occurs at $n=p$ and $m=q$, by updating sequentially, $p$ and $q$ are found as the smallest integers $n \geq 1$ and $m \leq n$, respectively.

For estimating the model parameters $\left\{\alpha_{i}, i=1 \rightarrow p\right\}$ and $\left\{\beta_{i}, i=0 \rightarrow q\right\}$ in (8), we let $r=p+q$ and derive $2 q$ linear equations that determine the $2 q$ unknowns coefficients $h_{k}$ 's and $g_{k}$ 's of the b.r. function $\rho_{p+q+1}(z)$ as in (17) such that the denominator polynomial of $H(z)$ is known completely. In matrix form, these equations become

$$
\mathrm{Ax}=\mathrm{b}
$$

where

$$
\mathbf{x} \equiv\left[\begin{array}{llllllll}
g_{1} & g_{2} & \ldots & g_{q} & h_{0} & h_{1} & \ldots & h_{q-1}
\end{array}\right]^{T},
$$

A is a $2 q \times 2 q$ matrix given by the coefficients $a_{k}$ 's of the error filter $E_{p+q}(z)$, i.e.,

$$
\begin{aligned}
& \mathbf{A} \equiv \\
& {\left[\begin{array}{cccccc}
a_{p} & \ldots & a_{p-q+1} & -a_{q} & \ldots & -a_{2 q-1} \\
a_{p+1} & \ldots & a_{p-q+2} & -a_{q-1} & \ldots & -a_{2 q-2} \\
\vdots & \ldots & \vdots & \vdots & \ldots & \vdots \\
a_{p+q-1} & \ldots & a_{p} & -a_{1} & \ldots & -a_{q} \\
a_{p+q} & \ldots & a_{p+1} & -1 & \ldots & -a_{q-1} \\
0 & \ldots & a_{p+2} & 0 & \ldots & -a_{q-2} \\
\vdots & \ldots & \vdots & \vdots & \ldots & \vdots \\
0 & \ldots & a_{p+q} & 0 & \ldots & -1
\end{array}\right]}
\end{aligned}
$$

and

$$
\mathrm{b} \equiv-\left[\begin{array}{llllllll}
a_{p+1} & a_{p+2} & \ldots & a_{p+q} & 0 & 0 & \ldots & 0
\end{array}\right]^{T} .
$$

The $\psi_{p+q+1}(z)$ in (39) can be identified in the algorithm of linear fractional transformations[6].

\section{Results and Discussions}

We generated a long sequence of data from an $\operatorname{ARMA}(4,2)$ model appeared in [1]

$$
\begin{aligned}
& H(z)= \\
& \frac{1.2345-1.0706 z+z^{2}}{1-2.7596 z+3.8078 z^{2}-2.6504 z^{3}+0.9224 z^{4}}
\end{aligned}
$$

which corresponds to two pairs of poles at $0.98 e^{ \pm j 0.22 \pi}$, $0.98 e^{ \pm j 0.28 \pi}$ and one pair of zeros at $0.90 e^{ \pm j 0.34 \pi}$, where the data length $N$ is 400 .

In Fig.3 (a), we show the result of the model order selection using the proposed scheme, and the accompanying line of dashes is of associated the criterion disscussed in [7], which is based on sample autocovariances $\hat{c}_{r}$ 's. To get a comparison, we also show the results of using Pillai's algorithm for model order selection in Fig.3 (b). Since the first stage where $\epsilon$ equals zero occurs at $n=p$ and $m=q$, sequential updating of $n$ and $m$ continues until substantial relative minima in the value of $\epsilon$ are observed to occur for the first time. The corresponding pair $(n, m)$ is then identified with $(p, q)$. With the proposed method, the curve indicates such a significantly substantial dip at the correct stage $n=4, m=2$.

The reconstracted model obtained from the proposed algorithm is given by

$$
\begin{aligned}
& \hat{H}(z)= \\
& 1-2.7968 z+3.8770 z^{2}-2.7080 z^{3}+0.9387 z^{4}
\end{aligned}
$$

and obtained from Pillai's algorithm is given by

$$
\begin{aligned}
& \hat{H}(z)= \\
& \frac{1.7685-1.5398 z+0.8165 z^{2}}{1-2.8148 z+3.8352 z^{2}-2.6239 z^{3}+0.8718 z^{4}}
\end{aligned}
$$

which spectra are shown in Fig.4. In addition, we selected 10 consecutive disjoint segments each with $N=400$ data and used each segment in simulations of the proposed method and Pillai's method, respectively. The corresponding pole-zero estimates are ploted as shown in Fig.5 (a) and (b). For the simulations of the proposed method, from Fig.5 (a), we see that the estimates of the zeros are somewhat conservative. This is because that, from (38), the MA estimates are related to $\sigma_{p+q}^{2}$, the prediction error power of a $(p+q)$ th-order lattice whitening filter, which is based on the estimated variance $\hat{c}_{0}$ of the $\operatorname{ARMA}(p, q)$ process $x(n)$. A similar discussion about the error of the MA estimates can 
be found in [9]. In the proposed method, model order selection and AR parameter estimation are completely independent of the estimates $\left\{\hat{c}_{r}\right\}$. And the simulation results indicated that our estimates are superior to, and are never poorer than Pillai's estimates based on sample autocovariance functions. The improvement in performance is due to the use of Burg lattice estimates.

\section{References}

[1] S.U. Pillai, T.I. Shim and D.C. Youla: A New Technique for ARMA-System Identification and Rational Approximation, IEEE Trans. Signal Processing, vol.41, no.3, pp.1281/1304 (1993)

[2] S.U. Pillai and T.I. Shim: Sepectrum Estimation and System Identification, New York, Springer-Verlag, N.T. (1993)

[3] A. Papoulis: Levinson's Algorithm, Wold's Decomposition, and Spectral Estimation, SIAM Review, vol.27, no.3, 405/441 (1985)

[4] B. Choi: ARMA Model Identification, New York, SpringerVerlag, N.T. (1992)

[5] J.D. Rhodes: Theory of Electrical Filters, New York, Wilsy (1976).

[6] T.T. Georgiou, and P.P. Khargonekar: Linear Fractional Transformation and Spectral Factorization, IEEE Trans. Automat. Contr., vol.AC-31, no.4, 345/347 (1986)

[7] J. Yao and S. Sugimoto: ARMA Modeling and Order Determination, Proc. of the 27th ISCIE International Symposium on Stochastic Systems Theory and Its Applications, Bappu, 113/119 (1995)

[8] J. Yao, K. Baku, and S. Sugimoto: Order Determination and Identification for Noisy AR Processes, Proc. of the 27th ISCIE International Symposium on Stochastic Systems Theory and Its Applications, Kyoto, 7/12 (1995)

[9] S. Li, and B.W. Dickinson: Application of the Lattice Filter to Robust Estimation of AR and ARMA Models, IEEE Trans. Acoust., Speech, Signal Processing, vol.36, no.4, $502 / 512$ (1988)

[10] P. Gahinet, and P. Apkarian: A Linear Matrix Inequality Approach to $H_{\infty}$ Control, Int. J. Robust \& Nonlinear Control, 4, 421/448 (1994)

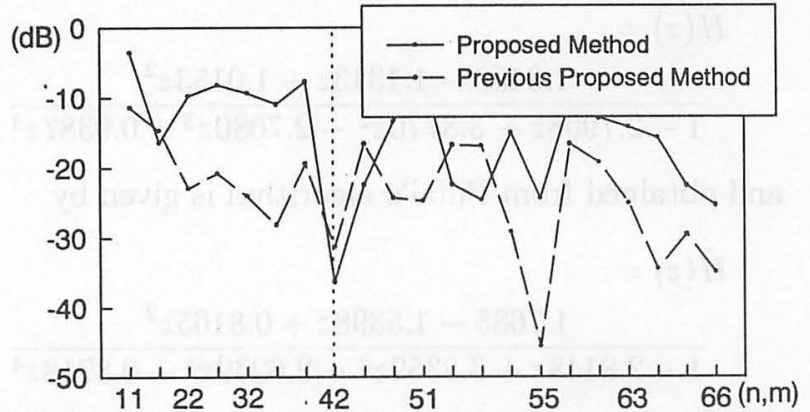

(a) Using the proposed method

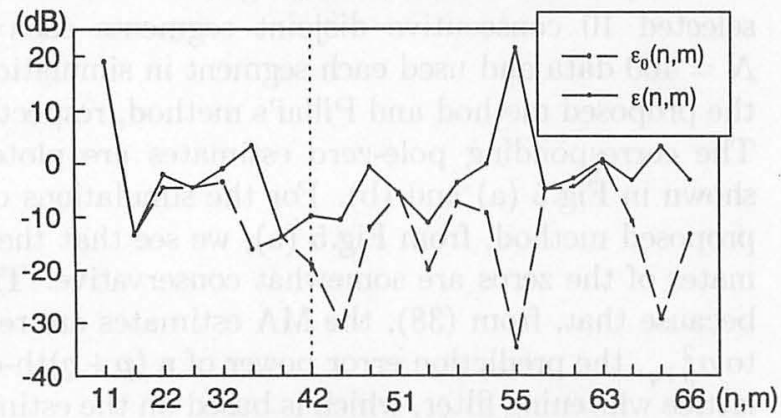

(b) Using Pillai's method

Fig. 3. Model order selection

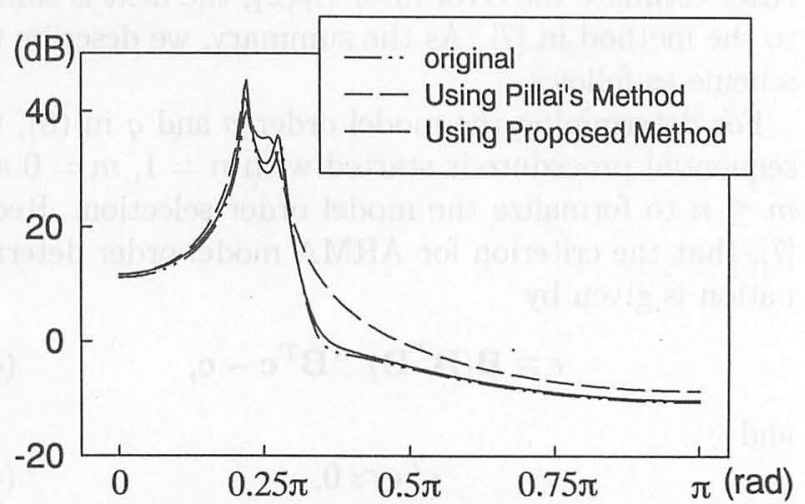

Fig. 4. Original and reconstructed spectra

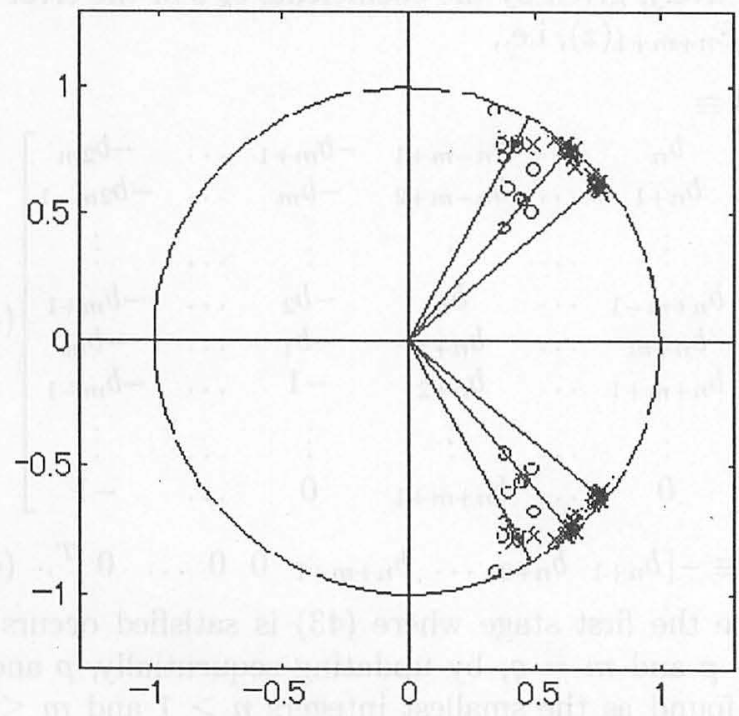

(a) Using the proposed method.

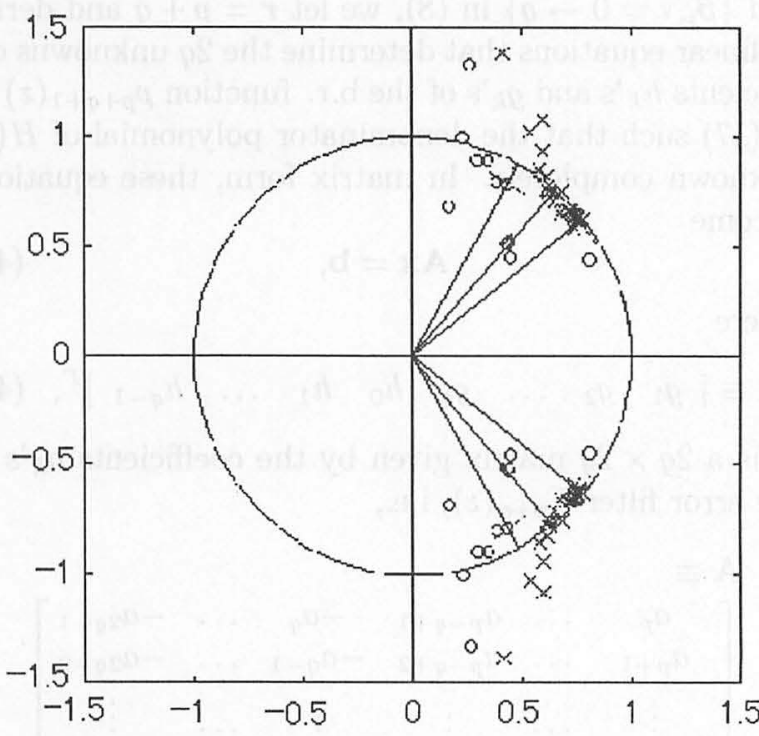

(b) Using Pillai's method

Fig. 5. Pole-zero estimates from 10 simulation results 